\title{
Variational design of smooth rational Bézier curves
}

\author{
Hans Hagen \\ Universität Kaiserslautern, FB Informatik, W-6750 Kaiserslautern. Germany
}

Georges-Pierre Bonneau

ENS Cachan, Paris, France

Received October 1990

Revised February 1991

Abstract

Hagen, H. and G.-P. Bonneau, Variational design of smooth rational Bézier curves, Computer Aided Geometric Design 8 (1991) 393-399.

'NURBS' are currently seen as the most promising curve form in CAD/CAM applications. Rational Bézier curves are special non-uniform rational B-splines. In this paper we describe a calculus of variation approach to design the weights of a rational curve in a way as to achieve a smooth curve in the sense of an energy integral.

Keywords. NURBS, rational Bézier curves, variational design, smoothing

\section{Introduction}

Curves and surfaces designed in a Computer Graphics environment have many applications, including the design of cars, airplanes, shipbodies and modeling robots. The generation of 'technical smooth' curves and surfaces (which are appropriate for the NC-process) from a set of two- or three-dimensional data points is a key problem in the field of Computer Aided Geometric Design.

One of the most promising curve- and surface-modelling methods is the NURBS-technique (NURBS - non-uniform rational B-splines). The fundamental idea of the rational Bézier- and $\mathrm{B}$-spline algorithms is to evaluate and manipulate the curves and surfaces by a (small) number of control points and weights.

The purpose of this paper is to present an algorithm to assign to the weights appropriate values to achieve technical smooth curves. The standard fairness criterion for curves in engineering is to minimize the strain energy in a thin elastic beam. The strain energy stored in the beam is proportional to the total curvature integral. We aproximate this integral by quadrature formulas. A calculus of variation approach based upon the integral criterions with respect to the weights of the rational curves, leads to a non-linear system of equations. This system is then solved by routines of the NAG-library. Several examples are presented to illustrate the concept. 


\section{Rational Bézier curves}

We assume that the reader is familiar with the concepts of non-rational Bézier- and B-spline curves (see [Farin '90] and [Hoschek \& Lasser, '89]). In this section we give the fundamental concepts of rational curves, following [Farin '89].

A rational Bézier curve of degree $n$ in $\mathbb{E}^{3}$ is the projection of an $n$th degree Bézier curve in $\mathbb{E}^{4}$ on to the hyperplane $w=1$.

$$
x(t)=\frac{w_{0} b_{0} B_{0}^{n}(t)+\cdots+w_{n} b_{n} B_{n}^{n}(t)}{w_{0} B_{0}^{n}(t)+\cdots+w_{n} B_{n}^{n}(t)}
$$

$x(t), b_{i} \in \mathbb{E}^{3} ; w_{i} \geqslant 0$ are called (scalar) weights.

We reparametrize a rational Bézier curve by changing the weights according to

$$
\tilde{w}_{i}:=c^{i} w_{i} ; \quad i=0, \ldots, n ; \quad c:=\left(w_{0} / w_{n}\right)^{1 / n}
$$

(see [Farin '89]) and get, after dividing all weights by $w_{0}$, the standard form

$$
x(t)=\frac{\sum_{i=0}^{n} w_{i} b_{i} B_{i}^{n}(t)}{\sum_{i=0}^{n} w_{i} B_{i}^{n}(t)} ; \quad w_{0}=w_{n}=1 ; \quad w_{i}>0 .
$$

This yields in the rational cubic case:

$$
x(t)=\frac{b_{0} B_{0}^{3}(t)+w_{1} b_{1} B_{1}^{3}(t)+w_{2} b_{2} B_{2}^{3}(t)+b_{3} B_{3}^{3}(t)}{B_{0}^{3}(t)+w_{1} B_{1}^{3}(t)+w_{2} B_{2}^{3}(t)+B_{3}^{3}(t)} .
$$

\section{Variational principles in curve design}

Free form objects are an essential part of powerful CAD systems. A major topic is the generation of smooth curves and surfaces which can be immediately supplied to the NC-process. The fundamental idea of our method is the use of modelling tools which minimize a certain functional that can be interpreted in the sense of physics and/or geometry. In the case of curves a thin elastic beam can serve as a model for a fair shape. Such a beam tends to take a position of least strain energy and the energy stored in the beam is proportional to the integral

$$
\int \kappa^{2}(t)\left\|x^{\prime}(t)\right\| \mathrm{d} t ; \quad \kappa(t) \text { is the curvature of the curve } x(t) .
$$

As an approximation of the integral criterion (3.1) we can use

$$
\int\left\|x^{\prime \prime}(t)\right\|^{2} \mathrm{~d} t \rightarrow \min .
$$

This criterion 'depends' on the parametrization. We consider cord length parametrization an appropriate choice for this kind of application.

Using higher derivatives a further smoothness criterion can be defined, which also has physical meaning.

In dynamics the quantity $R:=\mathrm{d} x / \mathrm{d} s=(\kappa(s) \cdot N(s))^{\prime}$ is called jerk and curves can be derived from the principle of jerk minimization

$$
\int\left\|x^{\prime \prime}(t)\right\|^{2} \mathrm{~d} t \rightarrow \min .
$$


The stiffness-degree concept (see [Hagen '90]) allows to combine (3.2) and (3.3) into one criterion:

$$
\int\left(\alpha\left\|x^{\prime \prime}(t)\right\|^{2}+\beta\left\|x^{\prime \prime \prime}(t)\right\|^{2}\right) \mathrm{d} t \rightarrow \min ; \quad \alpha, \beta \geqslant 0 ; \quad \alpha+\beta=1 .
$$

This means performing a blended optimization of energy and jerk. We adjust the $\alpha$ and $\beta$ in an interactive way. More research in this direction is under way. More material on variational principles in curve and surface design is in [Farin \& Hagen '90, Hagen \& Schulze '87, '90, Hagen '90, Nowacki \& Meier '87].

\section{Weight estimation for smooth curve design}

We consider (3.1) and (3.4) as fairness criteria ((3.2) and (3.3) are special cases of (3.4)) and approximate the integrals by quadrature formulas.

Neither the rectangle nor the Simpson-rule are appropriate for a calculus of variation approach. The resulting non-linear systems are of a too high degree. Therefore, we use the trapezoid rule

$$
\begin{aligned}
& \int_{a}^{b} \kappa^{2}(t)\left\|x^{\prime}(t)\right\| \mathrm{d} t \approx \frac{b-a}{2}\left(\kappa^{2}(a)\left\|x^{\prime}(a)\right\|+\kappa^{2}(b)\left\|x^{\prime}(b)\right\|\right), \\
& T E\left(w_{1}, w_{2}\right):=\frac{b-a}{2}\left(\kappa^{2}(a)\left\|x^{\prime}(a)\right\|+\kappa^{2}(b)\left\|x^{\prime}(b)\right\|\right),
\end{aligned}
$$

and for the stiffness-degree criterion we get

$$
\begin{aligned}
& \int_{a}^{b}\left(\alpha\left\|x^{\prime \prime}(t)\right\|^{2}+\beta\left\|x^{\prime \prime \prime}(t)\right\|^{2}\right) \mathrm{d} t \\
& \quad \approx \frac{b-a}{2}\left\{\alpha\left(\left\|x^{\prime \prime}(a)\right\|^{2}+\left\|x^{\prime \prime}(b)\right\|^{2}\right)+\beta\left(\left\|x^{\prime \prime \prime}(a)\right\|^{2}+\left\|x^{\prime \prime \prime}(b)\right\|\right)\right\} \\
& \quad=\operatorname{TS}\left(w_{1}, w_{2}\right) .
\end{aligned}
$$

A necessary condition for the energy minimum of a rational cubic curve is

$$
\frac{\partial T E}{\partial w_{1}}=0 \quad \text { and } \quad \frac{\partial T E}{\partial w_{2}}=0 .
$$

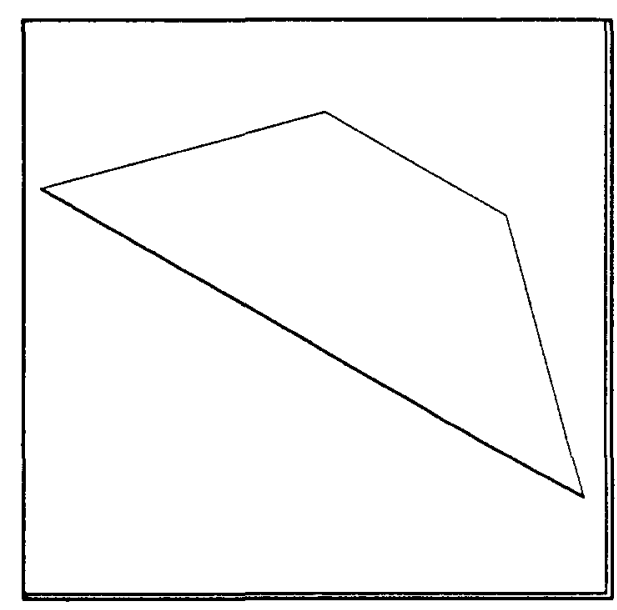

Fig. 1. 
Direct calculations yield a system for $w_{1}$ and $w_{2}$, which has only the trivial solution $w_{1}=0$ and $w_{2}=0$. In this case we have a straight line between $b_{0}$ and $b_{3}$ (see Fig. 1).

The stiffness-degree criterion

$$
\frac{\partial T S}{\partial w_{1}}=0 \quad \text { and } \quad \frac{\partial T S}{\partial w_{2}}=0
$$

leads to the following non-linear system

$$
\begin{aligned}
& {\left[\frac{(b-a)^{5}}{36}\right] \frac{\partial T S}{\partial \omega_{1}}\left(\omega_{1}, \omega_{2}\right)=3 \beta(\langle A, C\rangle+2\langle\tilde{B}, \tilde{C}\rangle)} \\
& \quad+\omega_{1}\left[(b-a)^{2} \alpha\left(\|A\|^{2}+\|\tilde{B}\|^{2}\right)+9 \beta\left(\|A\|^{2}-4\langle A, C\rangle+4\|\tilde{B}\|^{2}\right)\right] \\
& \quad+\omega_{2}\left[(b-a)^{2} \alpha(\langle A, B\rangle+\langle\tilde{A}, \tilde{B}\rangle)\right. \\
& \quad+9 \beta(2\langle A, B\rangle-\langle A, C\rangle-\langle B, C\rangle+2\langle\tilde{A}, \tilde{B}\rangle-\langle\tilde{A}, \tilde{C}\rangle-\langle\tilde{B}, \tilde{C}\rangle)] \\
& \quad+9 \omega_{1}^{2}\left[(b-a)^{2} \alpha\|A\|^{2}+9 \beta\left(-2\|A\|^{2}+\langle A, C\rangle\right)\right] \\
& \quad-6 \omega_{1} \omega_{2}\left[(b-a)^{2} \alpha\langle A, B\rangle+9 \beta\left(\|A\|^{2}+5\langle A, B\rangle+2\|\tilde{B}\|^{2}+2\langle\tilde{A}, \tilde{B})\right]\right. \\
& \quad-3 \omega_{2}^{2}\left[(b-a)^{2} \alpha\langle\tilde{A}, \tilde{B}\rangle+\beta\left(16\|B\|^{2}+18\langle A, B\rangle+8\|\tilde{A}\|^{2}+45\langle\tilde{A}, \tilde{B}\rangle\right)\right] \\
& +18 \omega_{1}^{3}\left[(b-a)^{2} \alpha\|A\|^{2}+54 \beta\|A\|^{2}\right] \\
& +486 \omega_{1}^{2} \omega_{2} \beta\left(\|A\|^{2}+2\langle A, B\rangle\right) \\
& +9 \omega_{1} \omega_{2}^{2} \beta\left(9\|A\|^{2}+8\|B\|^{2}+24\langle A, B\rangle+8\|\tilde{A}\|^{2}+9\|\tilde{B}\|^{2}+18\langle\tilde{A}, \tilde{B}\rangle\right) \\
& +36 \omega_{2}^{3} \beta\left(4\|A\|^{2}+9\langle\tilde{A}, \tilde{B}\rangle\right) \\
& -2430 \omega_{1}^{4} \beta\|A\|^{2} \\
& +973 \omega_{1}^{3} \omega_{2} \beta\left(\|A\|^{2}+\langle A, B\rangle\right) \\
& -27 \omega_{2}^{4} \beta\left(8\|\tilde{A}\|^{2}+9\langle\tilde{A}, \tilde{B}\rangle\right) \\
& +2187 \omega_{1}^{5} \beta\|A\|^{2}
\end{aligned}
$$

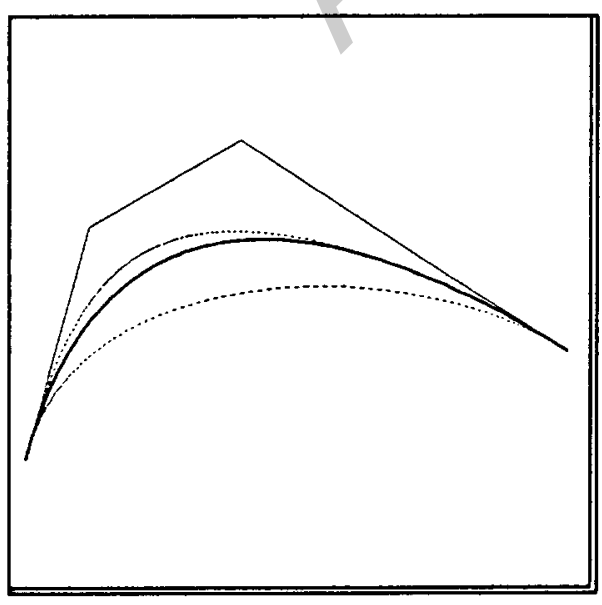

Fig. 2.
Example 1 (Fig. 2)

$\alpha=0.96$

$\begin{array}{lll}\omega_{1}=0.4 & \omega_{2}=0.3 & T S=56.44 \\ \omega_{1}=0.89 & \omega_{2}=0.84 & T S=35.41\end{array}$

$\omega_{1}=1.5 \quad \omega_{2}=1 \quad T S=2078.70$

Polynomial case

$\omega_{1}=1.0 \quad \omega_{2}=1.0 \quad T S=87.84$ 
with $A:=b_{1}-b_{0}, B:=b_{2}-b_{0} . C:=b_{3}-b_{0}, \tilde{A}:=b_{2}-b_{3}, \tilde{B}:=b_{1}-b_{3}, C:=-\tilde{C}$ and

$$
\begin{aligned}
& {\left[\frac{(b-a)^{5}}{36}\right] \frac{\partial T S}{\partial \omega_{2}}\left(\omega_{1}, \omega_{2}\right)=3 \beta(\langle\tilde{A}, \tilde{C}\rangle+2\langle B, C\rangle)} \\
& \quad+\omega_{1}\left[(b-a)^{2} \alpha(\langle\tilde{A}, \tilde{B}\rangle+\langle A, B\rangle)\right. \\
& \quad+9 \beta(2\langle\tilde{A}, \tilde{B}\rangle-\langle\tilde{A}, \tilde{C}\rangle-\langle\tilde{B}, \tilde{C}\rangle+2\langle A, B\rangle-\langle A, C\rangle-\langle B, C\rangle)] \\
& \quad+\omega_{2}\left[(b-a)^{2} \alpha\left(\|\tilde{A}\|^{2}+\|B\|^{2}\right)+9 \beta\left(\|\tilde{A}\|^{2}-4\langle\tilde{A}, \tilde{B}\rangle+4\|B\|^{2}\right)\right] \\
& \quad-3 \omega_{1}^{2}\left[(b-a)^{2} \alpha\langle A, B\rangle+\beta\left(16\|\tilde{B}\|^{2}+18\langle\tilde{A}, \tilde{B}\rangle+8\|A\|^{2}+45\langle A, B\rangle\right)\right] \\
& \quad-6 \omega_{1} \omega_{2}\left[(b-a)^{2} \alpha\langle\tilde{A}, \tilde{B}\rangle+9 \beta\left(\|\tilde{A}\|^{2}+5\langle\tilde{A}, B\rangle+2\|B\|^{2}+2\langle A, B\rangle\right)\right] \\
& +9 \omega_{2}^{2}\left[(b-a)^{2} \alpha\|\tilde{A}\|^{2}+9 \beta\left(-2\|\tilde{A}\|^{2}+\langle\tilde{A}, \tilde{C}\rangle\right)\right] \\
& +36 \omega_{1}^{3} \beta\left(4\|\tilde{A}\|^{2}+9\langle A, B\rangle\right) \\
& +9 \omega_{1}^{2} \omega_{2} \beta\left(9\|\tilde{A}\|^{2}+8\|\tilde{B}\|^{2}+24\langle\tilde{A}, \tilde{B}\rangle+8\|A\|^{2}+9\|B\|^{2}+18\langle A, B\rangle\right) \\
& +486 \omega_{1} \omega_{2}^{2} \beta\left(\|\tilde{A}\|^{2}+2\langle\tilde{A}, \tilde{B}\rangle\right) \\
& +18 \omega_{2}^{3}\left[(b-a)^{2} \alpha\|\tilde{A}\|^{2}+54 \beta\|\tilde{A}\|^{2}\right] \\
& +27 \omega_{1}^{4} \beta\left(8\|A\|^{2}+9\langle A, B\rangle\right) \\
& +972 \omega_{1} \omega_{2}^{3} \beta\left(\|\tilde{A}\|^{2}+\langle\tilde{A}, \tilde{B}\rangle\right) \\
& +2430 \omega_{2}^{4} \beta\|\tilde{A}\|^{2} \\
& +2187 \omega_{2}^{5} \beta\|\tilde{A}\|^{2} .
\end{aligned}
$$

This non-linear system was solved by the routine CO5 PBF of the NAG-library.

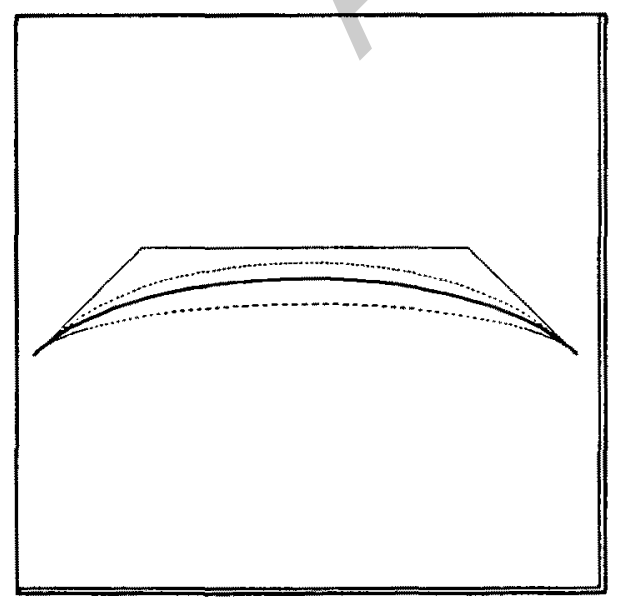

Fig. 3.
Example 2 (Fig. 3)

$$
\begin{array}{lll}
\alpha=0.5 & & \\
\omega_{1}=0.3 & \omega_{2}=0.3 & T S=1218.00 \\
\omega_{1}=0.82 & \omega_{2}=0.82 & T S=150.36 \\
\omega_{1}=2 & \omega_{2}=2 & T S=156550.00
\end{array}
$$

Polynomial case

$\omega_{1}=1.0 \quad \omega_{2}=1.0 \quad T S=378.00$ 


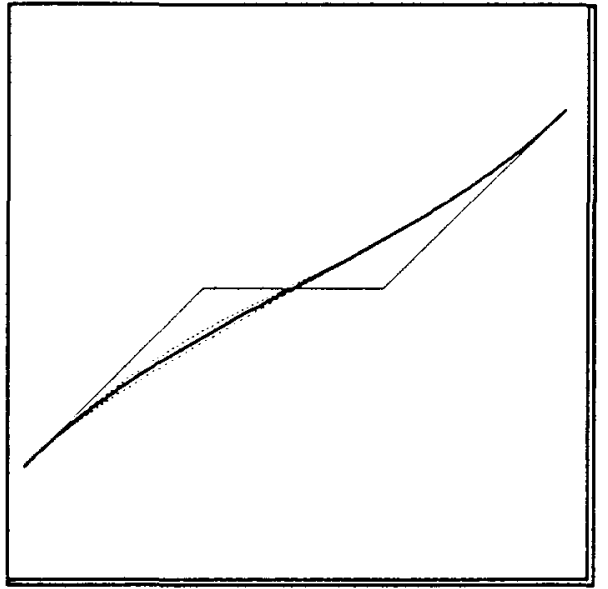

Fig. 4.

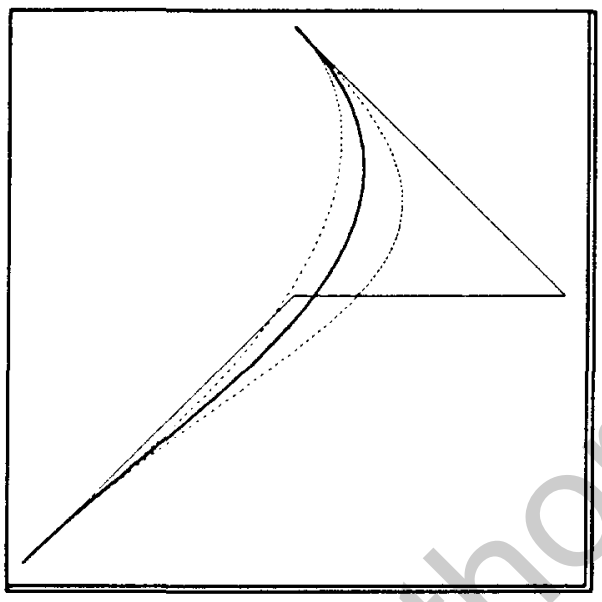

Fig. 5.
Example 3 (Fig. 4)

$\alpha=1$

$\omega_{1}=0.5$

$\omega_{1}=0.797$

$\omega_{2}=0.6$

$\omega_{2}=0.797$

$T S=23.32$

$\omega_{1}=1.2$

$\omega_{2}=1$

$T S=11.98$

$T S=117.88$

Polynomial case

$\omega_{1}=1.0 \quad \omega_{2}=1.0 \quad T S=36.00$

\section{Example 4 (Fig. 5)}

\section{$\alpha=1$}

$\omega_{1}=0.5 \quad \omega_{2}=0.3 \quad T S=7.32$

$\omega_{1}=0.655 \quad \omega_{2}=0.474 \quad T S=6.69$

$\omega_{1}=0.8 \quad \omega_{2}=0.9 \quad$ TS $=60.92$

Polynomial case

$$
\omega_{1}=1.0 \quad \omega_{2}=1.0 \quad T S=108.00
$$

\section{Examples}

The solid curves in the preceding examples are our optimal solution for each of the configurations.

\section{Conclusion}

A combination of energy and jerk minimization is used as a smoothing criterion. This criterion has a certain physical meaning. For this reason our algorithm is appropriate for NC-machining and 'Fair curve design'. Research concerning the generalization of this concept to surfaces is currently under way.

\section{References}

Farin. G. (1990), Curves and Surfaces for Computer Aided Geometric Design, Academic Press, New York. 2nd edition. Farin, G. (1989). Rational curves and surfaces, in: T. Lyche and L.L. Schumaker, eds., Mathematical Methods in CAGD. Academic Press, New York, 215-238. 
Farin. G. and Hagen. H. (1990), Local twist estimation, to be published in: H. Hagen. ed.. Curce and Surface Design. SIAM. Philadelphia. PA.

Hagen. H. (1990). Twist estimation for smooth surface design. to be published in the proceedings of the conference Mathematics of Surfaces, Bath (England).

Hagen. H. and Schuize. G. (1987), Automatic smoothing with geometric surface patches. Computer Aided Geometric 'Design 4 (3). 231-236.

Hagen. H. and Schulze. G. (1990), Variational principles in curve and surface design, to be published in: H. Hagen and Roller. eds., Geometric Modelling - Methods and Applications. Springer. Berlin.

Hoschek. J. and Lasser, D. (1989), Grundlagen der geometrischen Datenverarbeitung. Teubner. Leipzig.

Nowacki, H. and Meier. H. (1987), Interpolating curves with gradual changes in curvature. Computer Aided Geometric Design 4, 297-305. 